\title{
Feasibility and application of single-hole video-assisted thoracoscope in pulmonary peripheral tumors
}

\author{
XIN WANG, LEI WANG, HAO ZHANG, KE LI and XIANGNAN GONG \\ Department of Thoracic Surgery, The Central Hospital of Xuzhou, Xuzhou, Jiangsu 221009, P.R. China
}

Received December 29, 2015; Accepted August 26, 2016

DOI: $10.3892 / 01.2016 .5361$

\begin{abstract}
The feasibility and clinical application of single-hole video-assisted thoracoscope in pulmonary peripheral tumors was examined. From March, 2011 to March, 2015, we retrospectively analyzed the clinical data obtained from 32 patients with pulmonary peripheral tumor that received single-hole thoracoscopic surgery. We completed the surgery via a $1.5-\mathrm{cm}$ incision on the seventh or eighth rib in midaxillary line as the observation hole, and a 4.0-5.0-cm incision in the lateral margin of pectoralis major in the fourth or fifth rib in midaxillary line as the operation hole. All the patients had completed the tumor-reductive surgery under single-hole thoracoscope successfully. None required second operation hole or needed a transfer to thoracotomy. Operation time was 40-100 min with an average of $65.78 \pm 15.87 \mathrm{~min}$. Intraoperative blood loss was $20-100 \mathrm{ml}$, with an average of $47.19 \pm 26.91 \mathrm{ml}$. Post-operative chest drainage time was 3-6 days, with an average of 4.22 \pm 0.87 days. Hospitalization time after operation was 5-7 days, with an average hospitalization time of $5.97 \pm 0.82$ days. No patient received a second surgery for pulmonary leak or bleeding and no patient had any complication. All the cases recovered without any problem. In conclusion, for patients with pulmonary peripheral tumor, single-hole video-assisted thoracoscope could further reduce their surgical trauma. The operation was safe and feasible and worthy of wide application.
\end{abstract}

\section{Introduction}

In recent years, with the popularization of the concept of minimally invasive surgery and the application of video-assisted thoracoscopic surgery (VATS) technology, thoracic surgery has become a major surgical method in chest surgery. VATS incision has many options, and the most frequently used option is one observation hole and 2-3 operation holes. In recent years, with the development of VATS, a number of investigators have

Correspondence to: Dr Lei Wang, Department of Thoracic Surgery, The Central Hospital of Xuzhou, 199 South Jiefang Road, Xuzhou, Jiangsu 221009, P.R. China

E-mail: ks227h@163.com

Key words: single-hole, video-assisted thoracoscope, pulmonary tumor attempted to further reduce the operating hole on the basis of traditional thoracic surgery, only using a 4.0-5.0-cm incision as an operation (1). More minimally invasive operation, more appealing incision scar, more light postoperative pain, more rapid postoperative patient recovery are the prominent advantages (2). At present, this more minimally invasive surgery has been widely used in the treatment of primary spontaneous pneumothorax, lung cancer, mediastinal tumors and other diseases (3).

We performed a retrospective analysis of the efficacy of single-hole video-assisted thoracoscope in 32 pulmonary peripheral tumor patients that were treated at The Central Hospital of Xuzhou (Jiangsu, China) from March, 2011 to March, 2015. Summary studies are provided below.

\section{Materials and methods}

General materials. From March, 2011 to March, 2015, 32 patients, of whom were 22 males and 10 females, age range, 34-81 years (average, 55 years) were enrolled in the present study. All the patients were diagnosed with pulmonary peripheral tumor under CT examination. Tumors in 8 patients was located in upper left pulmonary lobe, 9 cases in left lower pulmonary lobe, 5 cases in right upper pulmonary lobe, and 2 cases in right middle pulmonary lobe, and 8 cases in right lower pulmonary lobe (Fig. 1). Eight patients were diagnosed with pulmonary malignant tumor before operation, but could not tolerate lobectomy operation due to pulmonary function impairment; 24 patients had no definite pathological diagnosis before operation, and confirmed to be pulmonary benign tumor after operation.

The present study was approved by the ethics committee of The Central Hospital of Xuzhou. Signed written informed consent was obtained from all the participants before the study

Anesthesia method and position. The patients underwent double-lumen tube intubation, intravenous combined anesthesia, and one-lung ventilation on healthy side during operation. Patients were required to lie on their healthy side and their upper arms in abduction position and fixed onto the hand bracket.

Operation method. Patients diagnosed with malignant lung tumor were confirmed to be unsuitable for lobectomy operation; thus, pulmonary wedge resection was carried out directly. 

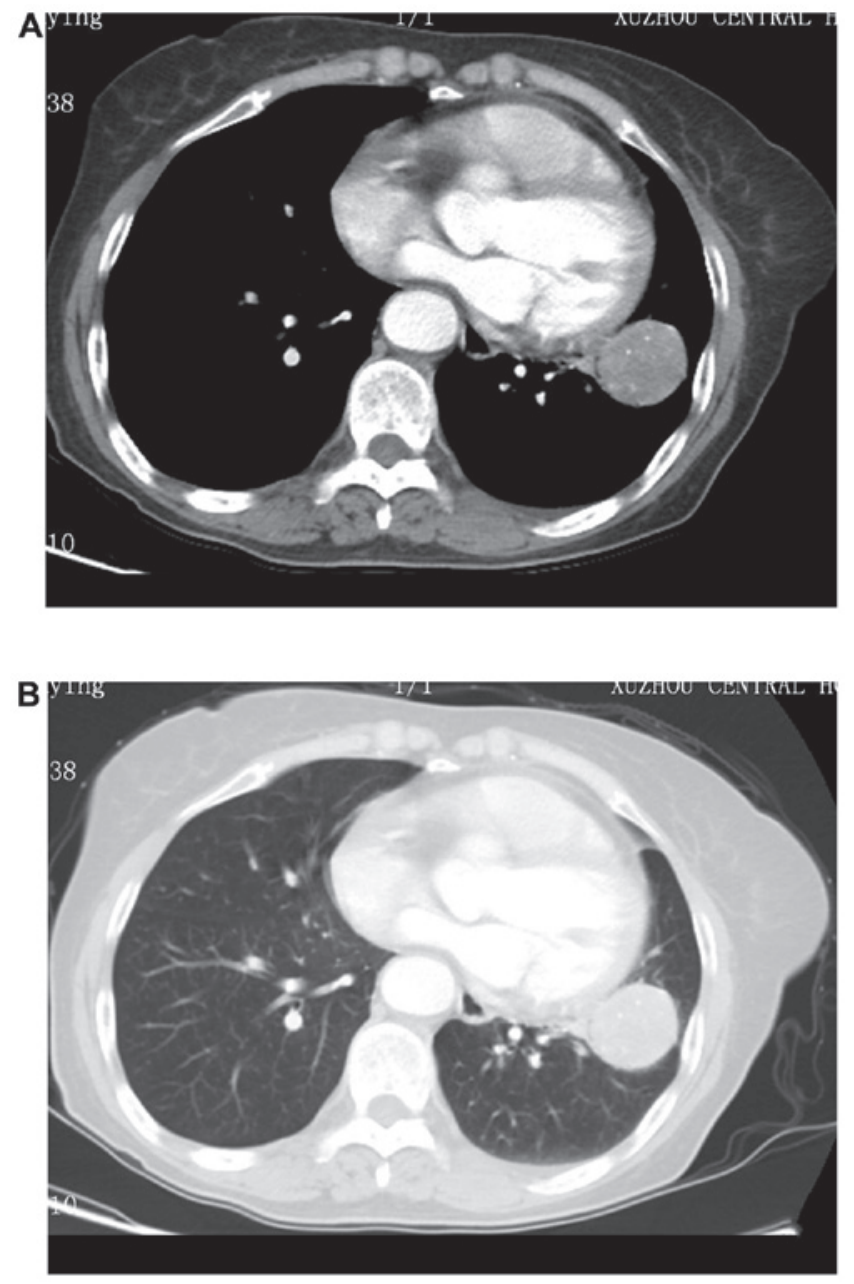
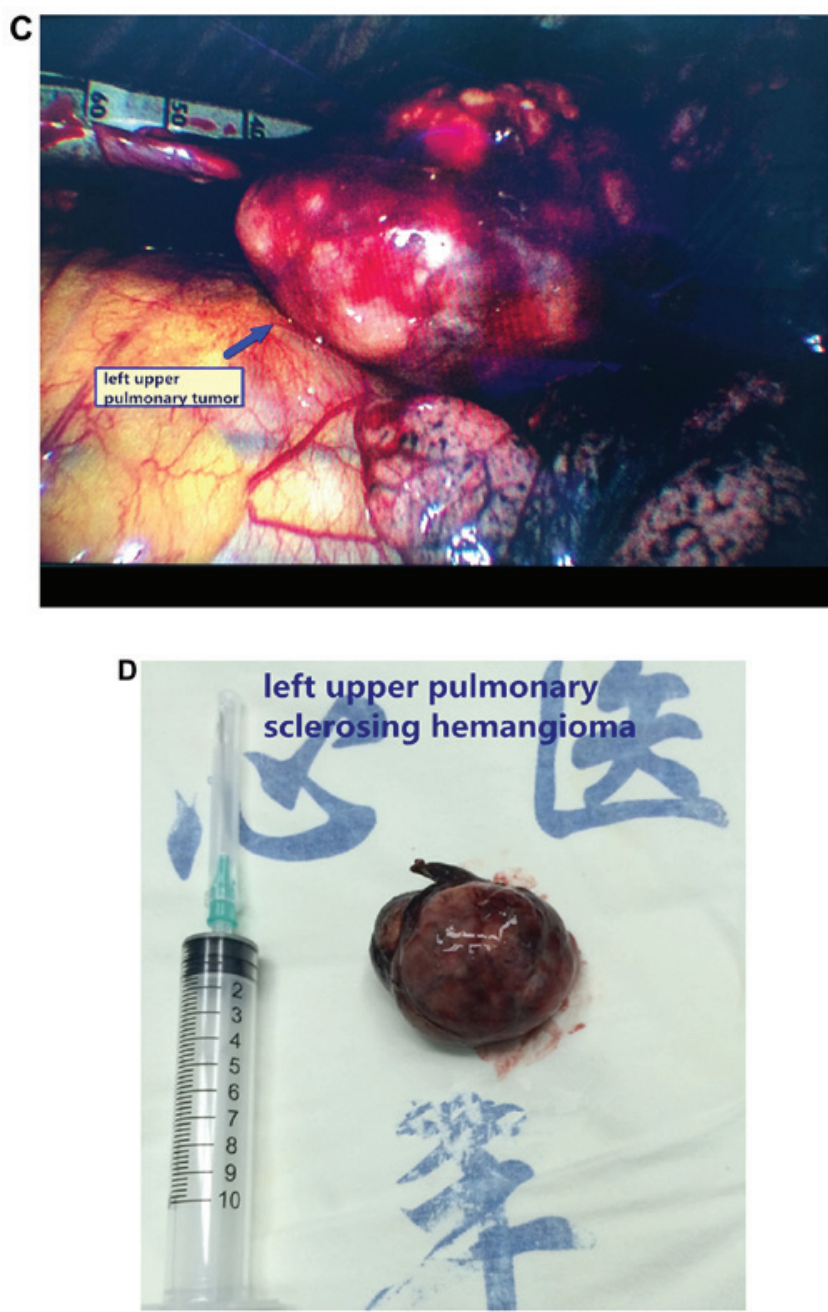

Figure 1. Images of typical cases. (A) Left upper pulmonary sclerosing hemangioma, (B) left upper pulmonary tumor, and (C and D) left upper pulmonary sclerosing hemangioma.

Those patients without pathological diagnosis before operation received pulmonary wedge resection and samples were sent for further pathological examination. When the pathological results confirmed the presence of benign pulmonary tumors, we terminated the operation. If pathological result confirmed the presence of malignant pulmonary tumors, we continued the pulmonary lobectomy and systematic lymph node dissection (patients were excluded from this study).

We used $30^{\circ} 10 \mathrm{~mm}$ thoracoscope and light source. During operation, the operator stood on the patient's abdomen side and the thoracoscope carrier was placed on the patient's back side. Initially, we made a $1.5-\mathrm{cm}$ incision on the seventh or eighth rib in midaxillary line as the observation hole, inserted the thoracoscope puncture trocar, and imbedded the thoracoscope to detect the whole chest cavity. We observed the tumor's location and condition of pleural adhesion. Subsequently we made a 4.0-5.0-cm incision in the lateral margin of pectoralis major in the fourth or fifth rib in midaxillary line as the operation hole and inserted an incision protective casing into the operation hole.

We palpated the tumor by thoracoscope surgical instruments or fingers to further determine the location and the size of tumor and used sponge forceps or endoscopic grasping forceps to pull the pulmonary lobe and exposed the surgery field. When the location of tumor was determined, we put in an endoscopic linear stapling device to make ' $\mathrm{V}$ ' type cutting alongside the tumor tissues and removed the tumor and partial lung tissues. While cutting, the tumors inside the lung were required to be completely enveloped within the normal lung tissues. Removed tumors were placed in extracting bags or gloves. After completing the resection, chest cavity was washed and examined for bleeding or leakage on the pulmonary cutting surface. When necessary, we used a 4-0 single absorbable suture material to suture the cutting surface. Meanwhile, our anesthesiologist made endotracheal sputum-suction to confirm whether there was any bloody discharge inside the patient's airway. As soon as the absence of bleeding or leakage was confirmed, the operation was terminated. Before closing the chest, we moved the thoracoscope into operation hole and under direct vision of thoracoscope we placed an intrathoracic drain into the observation hole. We sutured the operation hold by needle free suture unit or intradermal suture.

\section{Results}

All the patients completed the tumor resection smoothly under single-hole VATS. None of our patients required a second operation hole or needed a transfer to thoracotomy. Operation 
time was 40-100 $\mathrm{min}$, with an average of $65.78 \pm 15.87 \mathrm{~min}$. Intraoperative blood loss was $20-100 \mathrm{ml}$, with an average of $47.19 \pm 26.91 \mathrm{ml}$. Post-operative chest drainage time was 3-6 days, with an average of $4.22 \pm 0.87$ days. Hospitalization time after operation was 5-7 days, with an average of $5.97 \pm 0.82$ days. No patient received a second surgery for pulmonary leak or bleeding and none had any complications. All the patients recovered very well.

\section{Discussion}

Compared with traditional thoracotomy, VATS has many advantages, such as less trauma, less bleeding, and rapid recovery (4-6). After many years of clinical practice, many scholars have summarized the safety and reliability of complete VATS (7-9). Conventional VATS surgery usually requires 3 incisions, i.e., an observation hole in the 6th-8th rib in the midaxillary line, a main operator hole in the 3rd-5th rib in the anterior axillary line, and a deputy operation hole in the 6th-8th rib between the posterior axillary line and the scapular line. Occasionally, a third operation hole is required $(10,11)$. These three holes are distributed in a triangular shape $(10,11)$. Under 2-3 operation holes, the surgical device could enter into the chest cavity through different holes, which is convenient for the operation and also conductive to hemostasis, adhesion isolation and other operations. However, if surgical procedures could be improved and the number or size of operation holes could be reduced, it would be favorable for the optimization of VATS, making it less invasive, and thereby minimize the patient's trauma.

In 2004, Rocco et al reported the single-hole VATS wedge resection and confirmed that its operation results and post-operative complications were similar to three-hole surgery, but it could reduce the postoperative pain, shorten the duration of hospitalization, and reduce the overall cost (12). Based on these facts, our team improved the traditional VATS surgical incision design. By one operation hole in the anterior axillary line, we achieved favorable operation results. In the pressent study, all patients had completed the tumor-reductive surgery under single-hole thoracoscope successfully. None of the patients required a second operation hole or needed a transfer to thoracotomy and all patients had a good recovery. Chest back muscles have multiple layers, blood supply and a vast nerve distribution with narrow space between ribs. These characteristics make the incision in posterior axillary line more prone to bleeding and have more difficult homeostasis, which can translate to more pain for patients. Consequently clinicians abandoned the incision in posterior axillary line and simply made the operation hole in the anterior line axillary. Single-hole surgery could be completed by one surgeon and one thoracoscope assistant. Additionally, if during operation, patient needed a transfer to thoracotomy, single-hole surgery could also avoid excessive deputy operation holes, thereby reducing the scars. Nevertheless, single-hole surgery also has some drawbacks. Since the operation holes were reduced, the surgical instruments must enter and exit through the same hole. When several instruments are in use at the same time, they would interfere with each other. However, an experienced surgeon can manage this situation (13).
For some patients we used lung wedge resection, these included: i) patients with benign pulmonary tumors; ii) patients with malignant pulmonary tumors unsuitable for lobectomy; iii) patients with indefinite diagnosis; iv) with suspicious pulmonary metastatic nodules; and v) patients with the focus located in the periphery of the lung.

From our experience, we concluded the following notes for attention while performing single-hole VATS:

i) The hole should be closely stuck to the incision in upper edge of the rib and must be vertical. If the hole was tilted and formed a subcutaneous tunnel, the entry and exit for surgical instruments would be affected and also the operational angle for instruments would be limited (14).

ii) The single operation hole location should be determined by the site of the tumor and the size of incision should be suitable for tumor removal.

iii) Instruments with different length could be used to cooperate with the surgery. Moreover, we should make the most of the radian of instruments, and must avoid the instruments to interfere with each other (15).

iv) If necessary, the 'observation hole' and 'operation hole' could change their roles. Inserting an endoscopic linear stapling device through the observation hole could make the lung tissue resection more convenient.

v) Accurate localization of lung cancer was the key for a successful operation. For accurate localization we used the following methods: a) before surgery we injected methylene blue or edical glue into the focus through chest CT, or placing fine needle or steel wire to determine the position; and b) during operation we observed the pleural indentation in visceral layer of lung surface or navel signs; we made palpation by surgical instruments or single finger or double fingers; we performed B-ultrasonic localization during operation.

vi) We made a second operation hole or combine with small incision operation if during the operation one of the following circumstances occurred: a) the chest had extensive dense adhesion; b) the amount of bleeding was too large to be stopped; and c) air leakage from the lung was too serious and became inconvenient for suture. For the safety of patients, we should take other proper measures, instead of sticking to single hole surgery.

In conclusion, for patients with pulmonary peripheral tumor, single-hole video-assisted thoracoscope could further reduce their surgical trauma. The operation is safe, feasible and worthy of wide application.

\section{References}

1. Woo YJ and Reitz BA: One hundred years of history at Stanford University: thoracic and cardiovascular surgery. Semin Thorac Cardiovasc Surg 27: 388-397, 2015.

2. He CX: Professor Eugenio Pompeo: the present and future of minimally invasive thoracic surgery. Ann Transl Med 3: 344, 2015.

3. Bertoglio P, Davini F, Melfi FM and Mussi A: eComment. Marking techniques can increase the use of minimally invasive surgery for small and deep nodules of the lung. Interact Cardiovasc Thorac Surg 22: 207, 2016.

4. Yun L, Sui XZ, Jiang GC, Li JF, Liu J and Wang J: Experience of completely video-assisted thoracoscopic lobectomy in non-small cell lung cancer: series of consecutive 500 patients in single-center. Chin J Cardiov Surg 28: 3-6, 2012.

5. Nuo $X$ and Zhuo L: The clinical application of video-assisted thoracoscopic lobectomy. Chinese J Lung Dis 4: 57-59, 2011. 
6. Zhenyu S, Yong Z, Minwei G, Xiaofeng C, Qi S and Weimin Z: Clinical study on the treatment of non-small cell lung cancer by 3 kinds of surgical procedures. J Nanjing Med Univ 31: 427-430, 2011.

7. Xiandong T, Tiewen $\mathrm{P}$ and Bin W: Advantages of VATS in the treatment of non small cell lung cancer. Chin J Lung Dis 4: 33-37, 2011.

8. Guanchao J, Fan Y and Jun W: Progress of thoracoscopic lobectomy in the treatment of non-small cell lung cancer. Chin J Cardiovasc Surg 26: 291-293, 2010.

9. Li JF, Yang F, Li Y, Wang J, Liu J, Jiang GC, Zhao H, Liu YG, Zhou ZL and Bu L: Clinical analysis of 100 consecutive completely thoracoscopic lobectomy. Chin J Clin Thorac Cardiovasc Sur 16: 1-5, 2009

10. Jianxing H: Minimally invasive thoratic surgical procedures and atlas. Guangdong Sci Technol Press 1: 195-229, 2005.
11. Jun W: Chest disease VATS holo-authentic atlas. Jiangsu Sci and Technol Press, pp69-116, 2007.

12. Rocco G, Martin-Ucar A and Passera E: Uniportal VATS wedge pulmonary resections. Ann Thorac Surg 77: 726-728, 2004

13. Xin W, Lei W, Huayong W and Hao Z: Clinical analysis of single-hole VATS in the treatment of non-small cell lung cancer. Chin J Laparosc Surg 6: 26-29, 2013.

14. Che GW, Mei LY, Mei JD and Liu LX: Single utility port video-asslsted thoracoscopic surgery for 158 patients with pulmonary diseases. Chin J Clin Thorac Cardiovasc Surg 19: $116-119,2012$.

15. Xin W and Lei W: Treatment of single-hole VATS in primary spontaneous pneumothorax. Chin J Laparosc Surg 5: 45-47, 2012. 